\title{
PesQuisa em enfermagem: possibilidades da filosofia de Buber
}

\author{
Nursing research: possibilities of Buber's philosophy \\ Investigación en enfermeria: posibilidades de la filosofía de Buber
}

\author{
Stela Maris de Mello Padoin', Cristiane Cardoso de Paula', Diego Schaurich"I \\ 'Universidade Federal de Santa Maria. Faculdade de Enfermagem. Santa Maria, RS \\ "Universidade Federal do Rio Grande do Sul. Departamento de Enfermagem. Porto Alegre, RS
}

Submissão: 13/1 1/2008

Aprovação: 02/07/2009

\section{RESUMO}

As pesQuisas em Enfermagem têm colaborado para a construção do conhecimento próprio da profissão, sendo evidente a tendência Qualitativa, da Qual se destaca a abordagem fenomenológica. O presente artigo apresentará as possibilidades da filosofia de Martin Buber na pesquisa. Na fase de campo, pode subsidiar a ferramenta de entrevista, pois trata das relações e do diálogo. Na fase de análise, pode ser utilizado como referencial teórico para interpretação, pois apresenta subsídios para compreensão do encontro de presença entre os seres humanos, a partir do modo de ver a si e ao mundo. Assim, compreendemos a contribuição de Buber como referencial na produção Qualitativa em pesquisa.

Descritores: Pesquisa Qualitativa; Metodologia; Filosofia em Enfermagem.

\section{ABSTRACT}

The nursing researches have collaborated to build the profession knowledge. The tendency in Qualitative methodologies is evident, and among them we highlight the phenomenology approach. This paper will present the possibilities of Martin Buber's philosophy in research. In the field work, it can help the interview technieue because it is related with relationship and dialogue. During the analyses phase it should be applied as a theoretical framework to interpretation. The Martin Buber's philosophy present elements to understand the encounter of presence among the humans beings, it came from the way of see himself/herself and the world. Thus, we understand the Buber's contribution as a theoretical framework to data production in Qualitative research.

Descriptors: Qualitative research; Methodology; Philosophy Nursing.

\section{RESUMEN}

Las investigaciones en Enfermería tienen colaborado para la construcción del conocimiento propio de la profesión, siendo evidente la tendencia cualitativa, de la cual se destaca el abordaje fenomenológico. El presente artículo presentará las posibilidades de la filosofía de Martin Buber en la investigación. En la fase de campo, puede subsidiar la herramienta de entrevista, pues trata de las relaciones y del diálogo. En la fase del análisis, puede ser utilizado como referencial teórico para interpretación, ya Que presenta subsidios para comprensión del encuentro de presencia entre los seres humanos, a partir del modo de ver a si y al mundo. Así, comprendemos la contribución de Buber como referencial en la producción cualitativa en investigación.

Descriptores: Investigación Cualitativa; Metodología; Filosofía en Enfermería. 


\section{INTRODUÇÃO}

A enfermagem, ao longo do seu processo histórico-social, vem se consolidando como ciência, arte e profissão. Para isso, tem se utilizado das pesquisas para construção de um aporte de conhecimentos próprio.

O desenvolvimento de pesquisas em enfermagem, no Brasil, teve seu impulso a partir da criação dos cursos de pós-graduação stricto sensu, na década de 1970, Que foi garantida pela Lei da Reforma Universitária, $\mathrm{n}^{\circ} 5540 / 68^{(1)}$. A partir de 1980, houve a criação dos cursos de doutorado, Que possibilitou a ampliação e a consolidação das investigações. A partir da década de 1990, determinou-se a obrigatoriedade dos conteúdos de metodologia científica na graduação(2).

As primeiras peseuisas de enfermagem foram anteriores aos anos de 1990 e utilizaram, predominantemente, o método Quantitativo para discutir o cotidiano profissional - tendência coerente para época, pois o paradigma positivista ${ }^{(4)}$ foi dominante nas pesquisas da área da saúde, com destaque aos estudos experimentais ${ }^{(3)}$. Valorizaram-se a repetição, a observação dos fatos, a busca de explicações e causas, as técnicas de análise baseadas em números, a mensuração, a utilização de testes estatísticos e a linguagem matemática.

As preocupações estavam centradas no biologicismo das dificuldades ou facilidades para o autocuidado. A concepção vigente era do funcionamento bio-psíQuico do organismo humano, entendido como a soma de partes/órgãos ${ }^{(3)}$.

A partir da década de 1990, as pesquisas de Enfermagem mostram uma tendência Qualitativa, revelando progressivamente a supressão dos dualismos entre corpo e alma, entre o si mesmo (o eu) e o corpo, entre o sujeito e o objeto, entre o físico e o psíQuico ${ }^{(3)}$. Isso se evidenciou no modo de relação entre o pesquisador e o sujeito da pesquisa e na necessidade de compreender o ser do humano.

Atualmente, tanto a abordagem Quantitativa Quanto a Qualitativa têm sido empregadas, a partir de referenciais teórico-metodológicos, na busca de respostas para a complexidade do cotidiano assistencial $^{(5)}$.

As pesquisas de abordagem Qualitativa fundamentam-se em disciplinas das ciências da saúde e das ciências humanas. Destaca-se a contribuição da filosofia, especialmente com os referenciais da fenomenologia ${ }^{(6)}$.

A fenomenologia surgiu no final do século XIX, nascida das reflexões e dos estudos de Edmund Husserl e entendida como uma ciência voltada às experiências vividas, preocupada com a essência dos fenômenos existenciais do ser humano ${ }^{(7)}$. Compreende-se Que é um movimento Que busca a investigação, descrição, compreensão e interpretação dos fenômenos do existir, daQuilo Que se mostra a si e em si, tal como é, e é uma alternativa para trabalhos cuja abordagem traz consigo a necessidade de ver o outro, dentro do outro, como o outro $^{(8)}$.

Ao optar pelo referencial da fenomenologia, o pesquisador busca perceber-se sendo-no-mundo, perceber os outros e as relações. Suas inquietações emergem das suas experiências no mundo e estruturam-se em problema de pesquisa. As compreensões advindas do exercício de interpretação contribuem tanto para o corpo de conhecimento e prática da profissão, Quanto para a vida cotidiana. Assim, o referencial fenomenológico possibilita um modo de pesQuisar e um modo de $\operatorname{ser}^{(8-10)}$.

Como modo de pesquisar, a fenomenologia tem sido utilizada para compreender facetas do cuidado de enfermagem ${ }^{(1-13)}$. Tem possibilitado investigar o existir do humano nas dimensões da historicidade e da temporalidade, nas relações com o outro e com o mundo, a partir do seu modo de ser. Traz a possibilidade de refletir o cotidiano do mundo vivido ${ }^{(14)}$, nas dimensões objetivas e subjetivas no campo da saúde. Revelou-se como um caminho possível para a realização de investigações, vislumbrando um novo horizonte de compreensão da enfermagem ${ }^{(10)}$. No Brasil, adeuiriu notoriedade a partir do final da década de 1980, em meio a um movimento Questionador da aplicabilidade do método científico aos fenômenos humanos, sem discernir Que o objeto de pesquisa das ciências exatas era diferenciado daqueles das ciências humanas $^{(10)}$.

Tal movimento se reflete na construção de teorias, entre as Quais se destaca a Teoria de Enfermagem Humanística, colaborando na composição do corpo de saberes de enfermagem. Fundamentou-se na filosofia existencial e em pensadores como: Gabriel Marcel, Frederick Nietzsch e, principalmente, em Martin Buber ${ }^{(15-16)}$.

O presente artigo tem como objetivo apresentar possibilidades de aplicação do referencial fenomenológico de Martin Buber nas peseuisas de Enfermagem.

\section{REFERENCIAL FILOSÓFICO DE MARTIN BUBER}

A filosofia de Martin Buber está interessada no sentido da existência do ser do humano e traz para reflexão o compromisso com a experiência vivida ${ }^{(17)}$. Sua obra apresenta como fato primordial a relação entre os seres humanos e a palavra como sendo dialógica. Considera Que o diálogo não é entendido como uma categoria do raciocínio dedutivo, mas essencialmente um evento de presença ${ }^{(18)}$.

Buber entende que é por meio da palavra que o homem existe, uma vez Que é dialógica e contém o vivido. Compreende o homem como um ser de diálogo, Que se situa no mundo e se relaciona com os outros (seres e coisas). No mundo e diante dos outros, o homem evidencia a intencionalidade de suas atitudes por meio da dualidade: a relação EU-TU e o relacionamento EU-ISSO ${ }^{(19-20)}$.

A relação acontece Quando o EU (homem) se presentifica diante do TU (QualQuer outra pessoa, animal, coisa ou Deus), ou seja, o homem se apresenta na atitude de ser-com-o-outro e ser-com-omundo. O homem existe apenas no modo de ser-com, não há homem sozinho, independente; ele existe apenas na relação. Nesse encontro, ambos podem mostrar-se em sua totalidade e se reconhecer reciprocamente, instituindo um mundo compartilhado ${ }^{(18)}$.

O relacionamento EU-ISSO é outra possibilidade de o homem agir diante de outra pessoa ou coisa. No entanto, nesse modo de ser, o homem jamais estará em sua totalidade, pois essa atitude é de experimentação, objetivação e uso. O mundo do ISSO é o reino absoluto da causalidade ${ }^{(18)}$, correspondendo à ordenação científica, ao desenvolvimento da ciência.

A relação EU-TU e o relacionamento EU-ISSO são atitudes fundamentais à existência do homem. EU-TU representa um modo de encontro, através da atitude de relação entre dois seres Que estão disponíveis-para compartilhar algo e existir recíproca e mutuamente. EU-ISSO representa a construção e a elaboração do saber, do conhecimento, da ciência Que rege o mundo vivido e 
experienciado pelas pessoas ${ }^{(19)}$. Assim, o existir do humano se dá nessas relações/relacionamentos; no momento em Que uma acontece, a outra se torna latente, num movimento dinâmico em Que ambas se complementam ${ }^{(19)}$.

Para Buber, o diálogo se desenvolve no entre, ou seja, a palavra proferida pelo EU no encontro com o TU deixa de pertencer a ele e também não pertence ao outro, mas passa a localizar-se na relação EU-TU. O "entre" é o intervalo, o lugar de revelação da palavra proferida. Assim, a mola-mestra na interpretação da existência do homem é a 'relação', pois esta indica Que o significado desta existência não está nem nele, nem no mundo ou no outro, mas 'entre' os dois ${ }^{(18)}$.

Dessa forma, para Que os seres estabeleçam uma relação, há a necessidade da palavra, do diálogo inter-humano, Que permite a um EU presentificar-se no face-a-face com seu TU. Na relação inter-humana, é importante que cada um dos dois se torne consciente do outro, de tal forma Que, precisamente por isso, assuma para com ele um comportamento, Que não o considere nem trate dele como seu objeto, mas como seu parceiro num acontecimento de vida ${ }^{(21)}$.

Assim, a relação EU-TU é um encontro existencial em Que estão presentes: a reciprocidade, a intersubjetividade, o estar-com-ooutro em um mundo compartilhado. Essa atitude mostra um modo dialógico de ser e de existir, no Qual a palavra proferida pelo EU recebe a resposta do TU e, então, essa resposta é a responsabilidade do compartilhar. A relação EU-TU revela o voltar-se-para-o-outro, sendo, portanto, dialógica, ao passo Que o relacionamento EUISSO se desvela pelo dobrar-se-em-si-mesmo, sendo um modo monológico de $\operatorname{ser}^{(18)}$.

Portanto, a relação EU-TU, o relacionamento EU-ISSO, o diálogo, a intersubjetividade e o inter-humano são pressupostos Que poderão servir como subsídios para a enfermagem, em suas pesQuisas. Todos esses aspectos encontram-se interligados e são interdependentes, segundo a obra de Martin Buber.

\section{POSSIBILIDADES PARA A PESQUISA EM ENFERMAGEM}

Martin Buber apresenta um pensamento filosófico Que oscila entre o movimento fenomenológico e o existencialista, apresentando conceitos Que estão relacionados ao viver dos homens e às suas experiências na cotidianidade. Nesse artigo, vislumbra-se a aplicação dos conceitos EU-TU e EU-ISSO nos momentos da etapa de campo da pesquisa e de análise dos resultados, respectivamente.

$\mathrm{Na}$ etapa de campo, a relação EU-TU pode mediar as fases de ambientação no cenário, a seleção dos depoentes e a entrevista. Para iniciar essa etapa da pesquisa, faz-se necessária uma aproximação com o(s) cenário(s) no(s) Qual(is) o pesquisador coletará os dados para a investigação, favorecendo o contato e as articulações institucionais imprescindíveis ao desenvolvimento da pesquisa. Na ambientação, o pesquisador busca conhecer as pessoas e o cotidiano local. Nesse momento, o aspecto inter-humano e o diálogo permitem saber Quem são os possíveis sujeitos da pesquisa e selecionar aQueles Que mostram disposição-para participar das entrevistas.

Destaca-se a entrevista fenomenológica ${ }^{(22-24)}$, Que acontece como possibilidade de um encontro vivido e dialogado entre o pesquisador e o sujeito da pesquisa. Busca uma linguagem genuína, a fala originária $^{(19)}$ do sujeito da pesquisa, partindo de uma Questão norteadora, vinculada ao objeto de estudo. No decorrer do depoimento dialogado, o pesQuisador constitui Questões empáticas usando as próprias expressões da fala do sujeito de pesQuisa, aprofundando a compreensão do objeto de estudo da investigação.

Ressalta-se que, durante a entrevista, o EU (pesquisador) precisa liberar o olhar para a análise do vivido tal como ele se apresenta ${ }^{(23)}$, por meio do movimento de observar, captar, entender e compreender. O EU não deve estar fechado em uma perspectiva causal, mas, sim, atento ao dito e ao não-dito pelo TU (sujeito da pesquisa), sensível à sua expressividade e ao seu comportamento, como modos de mostrar-se. Esse é o momento de penetrar no mundo do TU, por meio do ouvir, olhar, envolver-se, pela fala, pelo silêncio e pelos gestos expressos na presença genuína do outro. O EU deixa-se impregnar por toda forma de dizer do TU, pensandoo não mais como pesquisador, mas com o próprio pensamento do sujeito da pesQuisa (sob a perspectiva própria do TU), abstraindose, assim, de todo e Qualquer pré-conceito.

Nesse encontro inter-humano, ambos passam a vivenciar sentimentos e emoções, em um mundo Que está sendo compartilhado. Nesse momento de intersubjetividade, o TU se mostra no movimento de pensar suas vivências e, concomitantemente, o EU reflete a si mesmo.

$\mathrm{Na}$ etapa de análise dos depoimentos gerados pelas entrevistas, o pesquisador assume a atitude EU-ISSO, distanciando-se da relação EU-TU. Ao adentrar no relacionamento objetivante, visa ao desenvolvimento da interpretação compreensiva daQuilo Que é o objeto e o objetivo da pesquisa. Isso acontece por meio da leitura daeuilo que o TU manifestou em sua totalidade e singularidade, não mais como um TU específico, mas como o ser do humano em busca de generalidade.

Assim, o modo EU-ISSO configura uma atitude de aproximação com o mundo do uso, da palavra obtida Que foi compartilhada no diálogo. O Que foi compartilhado é transcrito e mostra ao pesquisador as possibilidades de compreensão do vivido, a partir do Que foi desvelado e poderá ser analisado segundo as fases do método proposto na pesquisa e do referencial teórico, podendo ser, inclusive, o de Buber.

\section{CONSIDERAÇÕES FINAIS}

A filosofia de Martin Buber é um referencial teórico-filosófico possível de ser aplicado às pesquisas Qualitativas, especialmente àQuelas com abordagem fenomenológica. Isso se deve ao fato de o tema central de seu pensamento encontrar-se no vivido pelo homem, no sentido de sua existência e nos seus modos de ser.

O referencial buberiano mostra-se coerente com os princípios humanísticos do cuidado de enfermagem e, também, com seu objeto de estudo, dada à tendência de os pesquisadores de enfermagem voltarem seus olhares para as situações subjetivas dos seres humanos e do cuidado. Os resultados dessas pesquisas contribuem para a aproximação entre a teoria e a prática do cuidado, por meio da compreensão dos fenômenos Que permeiam o impacto da alteração ou não do estado de saúde das pessoas, das famílias e da sociedade.

Então, o referencial de Buber oferece possibilidades para o desenvolvimento da etapa de campo, subsidiada pela entrevista fenomenológica, contribuindo para a produção dos dados da pesquisa de enfermagem, pois trata das relações entre os seres 
humanos e das Questões do diálogo.

Também pode contribuir para a etapa de análise dos resultados, podendo ser utilizado como referencial teórico para a interpretação, pois apresenta subsídios para a compreensão do encontro de presença entre os seres humanos, a partir do modo de ver a si, aos outros (pessoas e coisas) e ao mundo.

\section{REFERÊNCIAS}

1. Ministério do Planejamento e Coordenação Geral (BR). Expansão do Ensino Superior e aumento de recursos para a Educação: relatório da Comissão Especial do Grupo de Trabalho da Reforma Universitária. Brasília: Ministério do Planejamento/ Ministério da Educação/Ministério da Fazenda; 1968.

2. Carvalho EC. A produção do conhecimento em enfermagem. Rev Latino-am Enfermagem 1998; 6(1): 119-22.

3. Capalbo C. Os novos paradigmas da pesquisa em saúde e a pós-modernidade. Rev Ciência Saúde 1997; 16(1-2): 16-31.

4. Rocha SMM, Silva GB. Linhas filosóficas e ideológicas na pesquisa em enfermagem no Brasil. Rev Bras Enferm 1987; 40(4): 214-21.

5. Prado ML, Gelbcke FL. Produção do conhecimento em enfermagem no Brasil: as temáticas de investigação. Rev Bras Enferm 2001; 54(1): 34-42.

6. Damaceno MMC, Lopes RLM. A apropriação da fenomenologia pela enfermagem: pesquisas fenomenológicas desenvolvidas por enfermeiras. Fortaleza: Pós-Graduação/Departamento de Enfermagem, Universidade Federal do Ceará; 1997.

7. Martins I, Boemer MR, Ferraz CA. A fenomenologia como alternativa metodológica para pesQuisa: algumas considerações. Rev Esc Enferm USP 1990; 24(1): 139-47.

8. Boemer MR. A fenomenologia na pesquisa em enfermagem. Seminário nacional de pesquisa em enfermagem; 1985; São Paulo (SP), Brasil. São Paulo: ABEn-CEPEn; 1985.

9. Boemer MR. A condução de estudos segundo a metodologia de investigação fenomenológica. Rev Latino-am Enfermagem 1994; 2(1): 83-94.

10. Corrêa AK. Fenomenologia: uma alternativa para a pesquisa em enfermagem. Rev Latino-am Enfermagem 1997; 5(1): 83-8.

11. Souza IEO, Paz EPA, Lopes RLM, Abreu ASGT, Camargo TC. Fenomenologia: moda ou modo de pesquisar e de compreender a realidade do cuidado de enfermagem. In: Macedo RMS, Berthoud CME. I ${ }^{\text {st }}$ Brazilian Internactional Conference on Qualitative Research: 24-27 março 2004. Taubaté (SP), Brasil.
Taubaté: Tec Art Editora e Núcleo de Pesquisa da Família; 2004.

12. Terra MG, Silva LC, Camponogara S, Santos EKAS, Souza AIJ, Erdmann AL. Na trilha da fenomenologia: um caminho para a pesquisa em enfermagem. Texto Contexto Enferm 2006; 15(4): 672-8.

13. Silva JMO, Lopes RLM, Diniz NMF. Fenomenologia. Rev Bras Enferm 2008; 6I (2): 254-7.

14. Stein E. Mundo vivido: das vicissitudes e dos usos de um conceito da fenomenologia. Porto Alegre: EDIPUCRS; 2004.

15. Paterson J, Zderad L. Enfermería humanistica. Cuidad de México: Limusa; 1979.

16. Paula CC, Schaurich D, Padoin SMM, Crossetti MGO. O cuidado como encontro vivido e dialogado na Teoria de Enfermagem Humanística de Paterson e Zderad. Acta Paul Enfermagem 2004; 17(4): 425-31.

17. von Zuben NA. Introdução. In: Buber M. Eu e tu. $5^{\mathrm{a}}$ ed. São Paulo: Moraes; 1977. p. 5-28.

18. Buber M. Eu e tu. $5^{\text {a }}$ ed. São Paulo: Moraes; 1977.

19. Schaurich D, Padoin SMM, Motta MGC. Experienciando um marco conceitual para o cuidado em enfermagem ao ser-familiar e/ou cuidador e ao ser-criança. Cogitare Enferm 2003; 8(2): 34-43.

20. von Zuben NA. Martin Buber: cumplicidade e diálogo. Bauru: EDUSC; 2003.

21. Buber M. Do diálogo e do dialógico. São Paulo: Perspectiva; 1982.

22. Carvalho AS. Metodologia da entrevista: uma abordagem fenomenológica. Rio de Janeiro: Agir; 1987.

23. Capalbo C. Prefácio. In: Carvalho AS. Metodologia da entrevista: uma abordagem fenomenológica. Rio de Janeiro: Agir; 1987. p. 5-8.

24. Simões SMF, Souza IEO. Um caminhar na aproximação da entrevista fenomenológica. Rev Latino-am Enfermagem 1997; 5(3): 13-7. 